\title{
A Survey of Alzheimer's disease using Fuzzy Neural Network
}

\author{
BEHNAZ Ghorbani ${ }^{1}$, MANOUCHEHR Kazemi ${ }^{2}$, MAHDIEH Nadi Senejani ${ }^{3}$ \\ 1, 2, 3 Department of Computer Engineering, Ashtian Branch, Islamic Azad University \\ Ashtian, Markazi, Iran
}

\begin{abstract}
Alzheimer disease is one of the most common diseases in the aging population, ranking as the fourth most common cause of death and it is a progressive neurodegenerative disorder characterized by the gradual onset of dementia. Magnetic resonance imaging (MRI) is a preferred neuro imaging examination for Alzheimer disease diagnosis. It allows accurate 3dimensional (3D) volume measurement of brain structures. The primary role of MRI in the diagnosis of Alzheimer disease is the assessment of volume change in characteristic locations. The diagnosis should be made on the basis of two features:

mesial temporal lobe atrophy and temporoparietal cortical atrophy.

Computer implementations increased the accuracy and performance of detecting undergoing changes in affected brain areas. It uses Image Segmentation and integrating of its algorithms provides simpler guidance for the diagnosis process. This paper provides a survey of existing techniques of image segmentation and a comparison of the K-means and neuro-fuzzy algorithms are also given.
\end{abstract}

Keywords: Image Segmentation; neuro-fuzzy; K-means; Alzheimer; MRI.

\section{Introduction}

The brain is the command centre which responsible for controlling diverse functions related to body movement, blood flow, cardiac activities, body temperature, emotional behavior, learning and memorization and so on. Despite intensive research, the classification of MRI data in terms of normal and abnormal brain tissues for performing fast and accurate diagnostics remains challenging [6]. Segmentation of brain for abnormality detection in slice image is the most daunting task due to its complex anatomy and problems inherent to the nature of the image. The heterogeneous and diffuse manifestation of pathology in medical images often prohibits the

\footnotetext{
${ }^{1}$ E-mail address: behnaz.ghorbani@gmail.com

${ }^{2}$ E-mail address(Corresponding author): univer_ka@yahoo.com \& m.kazemi@aiau.ac.ir

${ }^{3}$ E-mail address: nadi.mahdieh@aiau.ac.ir
} 
employment of computational methods. Furthermore, all imaging modalities carry limitations and artefacts which must be addressed and solved by segmentation methods. In this study the brain images are selected for the image reference as the injuries or abnormalities which can affect the large part of an organ.

Table 1 shows a simplified classification of computer vision tools. Segmentation is one of them.

Table 1: A Simple Classification of Computer Vision Tools [3]

\begin{tabular}{|l|l|}
\hline \multicolumn{1}{|c|}{ Basic Tools } & \multicolumn{1}{c|}{ Example Application } \\
\hline Filters & $\begin{array}{l}\text { noise suppression } \\
\text { edge detection } \\
\text { texture description }\end{array}$ \\
\hline Segmentation & $\begin{array}{l}\text { object recognition } \\
\text { image retrieval } \\
\text { medical image analysis }\end{array}$ \\
\hline Interest operators & $\begin{array}{l}\text { image matching } \\
\text { motion analysis } \\
\text { object recognition } \\
\text { image retrieval }\end{array}$ \\
\hline Photogrammetric operations & 3D reconstruction \\
\hline
\end{tabular}

Segmentation operators partition an image into none overlapping regions, each of them is homogeneous in one or more features and maximal in terms of this homogeneity.

Section 1.1 provides a summary of the work done. Section 1.2 presents an overview of image segmentation and segmentation techniques. Section $1.3 \& 1.4$ compare K-means and Neuro-fuzzy techniques.

\subsection{Related Works}

MRI helps physicians to diagnose brain diseases by identifying abnormalities in the brain images. This process suffers from taking a long time and also increasing the number of images, reduced sensitivity of the human eye, especially when the affected area is small slices of the brain contains information. Therefore, the MRI classifications are proposed. MR images include both normal and abnormal slices.

Clarke et al. (Clark et al., 1994) identified the abnormality in MR images by fuzzy c-means (FCM) algorithm. Antonie (Antonie, 2008) introduced a method for automated segmentation and categorization of brain MR images using SVM classifier [6]. Osama (2008) explained about the comparison of clustering algorithms and its application based on the type of dataset used.

In 2009, Stefan et al. described the structured patient data for the analysis of the implementation of a clustering algorithm. 
Klein et al.(Klein etal., 2010) used the dissimilarity matrix, a k-nearest neighbors (k-NN) classifier to detecting a premature dementia without using rigid registration of MRI [6].

Ketan Machhale et al. (2015) proposed an intellectual classification system to recognize the normal and abnormal MRI brain images.

A lot of works on certain varieties of constrained clustering have been done in the literature (Gordon, 1973; Ferligoj \& Batagelj, 1983; Lefkovitch, 1980). Thompson and Langley (1991) performed experiments with providing an initial "priming" concept hierarchy to several incremental unsupervised clustering systems.

Additionally, Talavera and B'ejar incorporated domain knowledge into an agglomerative algorithm, ISAAC (Talavera \& Bejar, 1999). It is possible for the k-means algorithm to evolve empty clusters in the course of its iterations. This is undesirable, since it can produce a result with fewer than k clusters.

Swarnalatha et al. (2013) proposed a concept based on the novel fuzzy approach with bit plane FCMBP approach.

Walaa Hussein Ibrahim et al. (2013) proposed the Neural Network techniques for the classification of magnetic resonance brain images. This work consists of three stages, preprocessing, dimensionality reduction, and the classification.

Fourier series are used to extract the exact information of boundary region [9].

Fourier descriptors are utilized for precise extraction of boundary features of the diseases region. As Fourier Descriptors introduce a large number of feature vectors that may invite the problem of over learning and chance of misclassifications, the proposed diagnosis system efficiently search the significant boundary features by genetic algorithm and feed them to the adaptive neuro-fuzzy based classifier.

\section{Segmentation Techniques}

\subsection{Overview Of Segmentation}

Image segmentation is one of the most critical tasks in automatic image analysis. It consists of subdividing an image into its constituent parts and extracting these parts of interest (objects).

Detection and correct segmentation of brain image is very difficult and challenging task due to low contrast image, unknown noise, unknown shape and size of brain which varies from patient to patient, partial volume effect caused because of artefacts and uneven texture of tumours which appear differently for different patients. 
Analysis of brain MRI includes various phases as shown in Figure 1[7] where images are first pre-processed and enhanced mainly to remove noise, normalize intensity values, improve contrast and extract brain from skull. Pre-processing helps in separating desired regions more accurately and also improves classification accuracy.

After pre-processing, images are divided into homogeneous regions with similar properties called segmentation and features are extracted in feature extraction step. Most commonly seen Pattern Recognition problem in the domain of medical image analysis is finding discriminating features from a set of medical images and classifying them into set of classes.

Feature extraction and feature selection and classification of brain lesions detected a very important role to play. Features are extracted mainly based on intensity values, texture properties, intensity gradients and edge detection.

Important features are selected in the feature selection phase by applying algorithms and based on these selected features. This procedure is shown in Figure 1 [7].

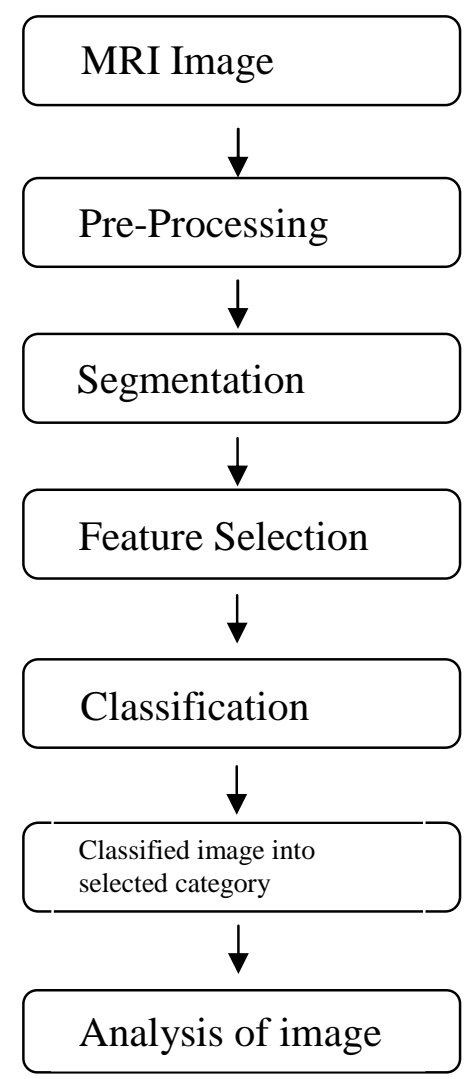

Fig.1: Block diagram of brain MRI classification and analysis 


\subsection{Segmentation Techniques}

A brief review on MRI segmentation methods and algorithms are discussed in the following [1].

a. Thresholding

In Thresholding technique, image is segmented by comparing pixel values with the predefined threshold limit. The image is partitioned directly into different regions based on the intensity values.

$f(x, y)$ be the input image and ' $T$ ' be the threshold value then the segmented image $g(x, y)$ is given by

$$
g(x, y)=\left\{\begin{array}{l}
1, \text { if } f(x, y)>T \\
0, \text { if } f(x, y) \leq T
\end{array}\right.
$$

b. Edge based segmentation

In this technique an image is segmented based on rapid changes in intensity near edges. Gray histogram and gradient based method are two main edge based segmentation methods.

c. Region based segmentation

In this technique an image is segmented into regions that are similar according to a set of predefined criteria. Segmentation algorithms based on region mainly include following methods:

1- Region growing

2- Region splitting and merging

3- Watershed segmentation

4- Snakes

5- Level Set Model

d. Classifiers

KNN- K-Nearest Neighbour ( $\mathrm{k}-\mathrm{NN}$ ) classification technique is based on a distance function and a voting function.

SVM- Support Vector Machine is a supervised classifier with associated learning algorithm derived from the statistical theory. It was first developed as an extension of the Generalized Portrait algorithm.

PCA- Principal Component Analysis (PCA) is used to extract principal features. PCA is mainly used to reduce the large dimensionality of the data by removing the redundant features. 
e. Clustering

This technique classifies pixels into classes, without knowing previous information or training.

1- K-means Clustering

2- Fuzzy C-means (FCM)

3- Expectation maximization

4- SOM

5- Hierarchical clustering

6- Ant tree algorithm

f. Artificial Neural Network

In this technique an image is firstly mapped into a Neural Network. Neural network segmentation includes two important steps feature extraction and image segmentation based on neural network.

1- Feed Forward Network

2- Feed Back Network

g. Hybrid

Table 1: A Summarized Diagram of Classification's methods

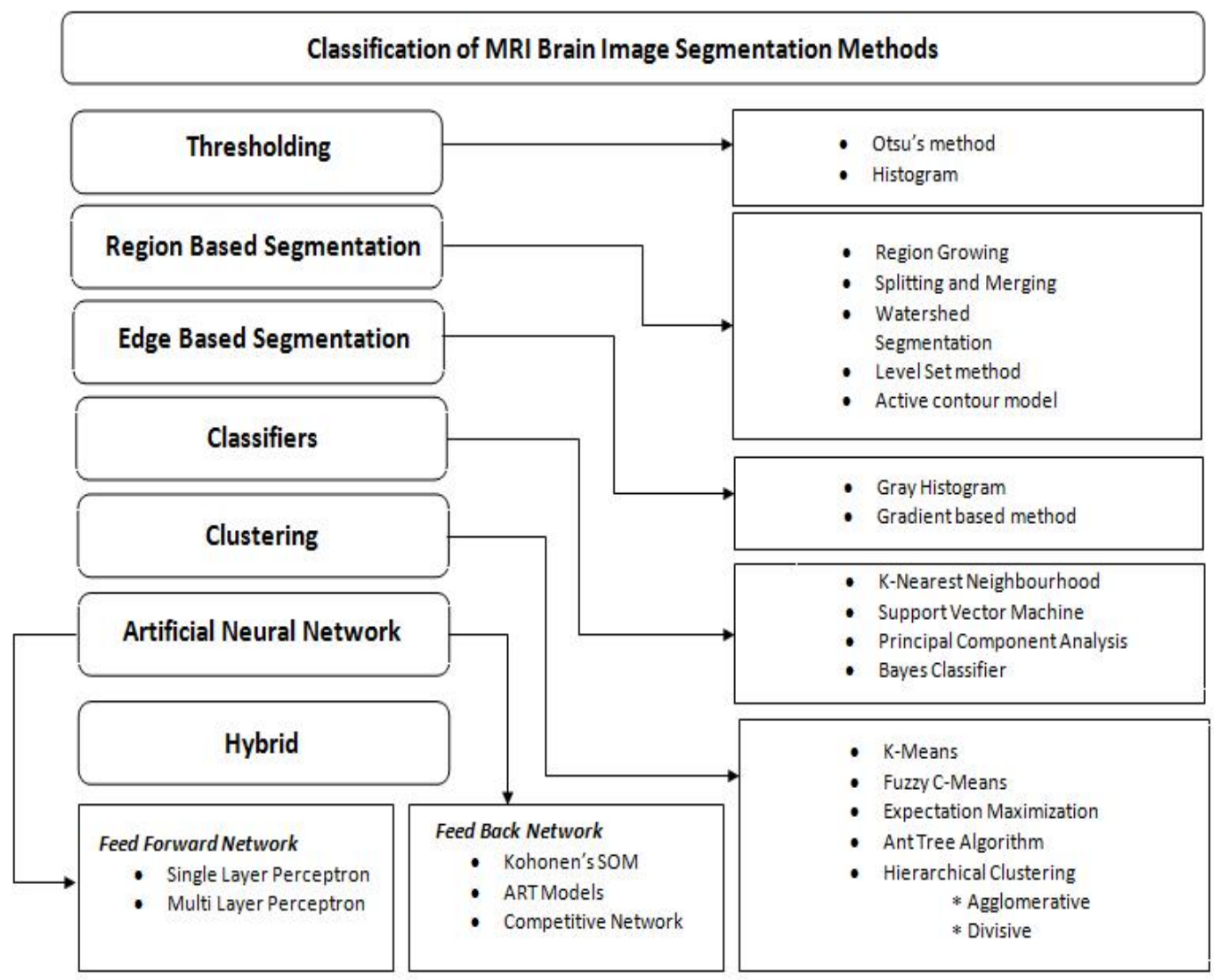




\section{K-Means}

This algorithm solves the well-known clustering problem. The procedure follows an easy way to classify a given data set through a different number of clusters. K-means is a well known partitioning method.

The K-means clustering and boundary detection techniques have been applied to extract the brain region (such as Alzheimer atrophy).

In K-means ' $\mathrm{K}$ ' centres are defined, one for each cluster. These clusters must be placed far away from each other. The next step is to take a point belonging to a given data set and associate it to the nearest centre.

When no point is pending, the first step is completed and early grouping is done. The second step is to recalculate ' $\mathrm{k}$ ' new centroids as barycentre of the clusters resulting from the previous step. After having ' $\mathrm{K}$ ' new centroids a new binding has to be done between the same data set points and the nearest new centre. A loop has been generated. As a result of this loop, the k centres change their location step by step until centres do not move any more. Finally this algorithm aims at minimizing an objective function known as squared error function given by [1]:

$$
J(V)=\sum_{i=1}^{c} \sum_{j=1}^{c_{i}}\left\|x_{i}-V j\right\|^{2}
$$

$\| \mathrm{x}_{\mathrm{i}-\mathrm{V}_{\mathrm{j}} \|:}$ the Euclidean distance between $\mathrm{xi}$ and $\mathrm{vj}$

' $\mathrm{Ci}$ ' is the number of data points in ith cluster.

' $\mathrm{C}$ ' is the number of cluster centres.

Algorithmic steps for K-means clustering:

$X=\{x 1, x 2, x 3 \ldots x n\}$ be the set of data points and $\mathrm{V}=\left\{\mathrm{v}_{1}, \mathrm{v} 2, \mathrm{v} 3 \ldots \mathrm{vc}\right\}$ be the set of centres.

Step1: Randomly select 'c' cluster centres

Step2: Calculate the distance between each data point and cluster centres.

Step3: Assign the data point to the cluster centre whose distance from the cluster centre is the minimum of all the cluster centres.

Step4: Recalculate the new cluster centre using

$$
v_{i}=\left(\frac{1}{c_{i}}\right) \sum_{j=1}^{c_{i}} x_{i}
$$


Where ' $\mathrm{Ci}$ ' represents the number of data points in ith cluster.

Step5: Recalculate the distance between each data point and newly obtained cluster centres.

Step6: If no data point was reassigned then stop, otherwise repeat from step 3.

K-means algorithm is fast and easier to understand. It gives a good result where you have separated data set from each other. But, if there are overlapping data then k-means will not be able to resolve as well.

\section{Neuro-Fuzzy}

In a new approach for classification of MRI images using a fuzzy inference system is proposed. Neuro-Fuzzy Logic explores approximation techniques from neural networks to find the parameter of a fuzzy system.

Neuro-fuzzy is the hybrid of the neural networks and fuzzy logic. It uses the advantages of both systems while reducing their disadvantages.

Fuzzy Neural Network or Neuro-Fuzzy system is a learning machine which uses the data from a fuzzy system. It does this by exploiting approximation techniques from neural networks. It eliminates the individual weaknesses of neural network and fuzzy logic while making use of their best advantages.

Fuzzy Logic (FL) is a branch of machine intelligence (Artificial Intelligence) that helps computers paint vivid pictures of the uncertain world. Fuzzy sets were introduced as a means of representing and manipulating data that are not precise, but rather fuzzy. The theory of fuzzy logic provides a mathematical strength to capture the uncertainties associated with human cognitive processes, such as thinking and reasoning. Fuzzy logic technique is more accurate but it fully depends on expert knowledge.

In standard set theory, an object does or does not belong to a set. There is no middle ground. In such bivalent systems, an object cannot belong to both its set and its compliment set or to neither of them. This principle preserves the structure of the logic and avoids the contradiction of object that both is and is not a thing at the same time. Fuzzy logic is highly abstract and employs experiment requiring human experts to discover rules about data relationship.

Fuzzy neural approach found to have more accurate decision making as compare to their counterparts. The obtained features are processed using fuzzy classification layer before passing it to neural network. Because of overlapping in medical images, it is possible that a pixel may belong to various clusters with different degree of membership which fuzzy if then else rules are applied to develop a classifier. 
Neural networks have the capability of efficiently learning the behaviour of a system and it can successfully generate the fuzzy "if then else rules" and fuzzy membership functions.

Types of Neuro-Fuzzy Systems:

The categories of different combinations of Neuro-Fuzzy techniques are as follows [14].

1- Cooperative Neuro-Fuzzy System

2- Concurrent Neuro-Fuzzy System

3- Hybrid Neuro-Fuzzy System

\section{Conclusion}

The Neuro-Fuzzy classification as a main classifier helps to divide the images into normal and abnormal. Neuro-fuzzy system presents solutions to process MRI images and segmenting abnormal images.

Neuro-fuzzy modeling is known as a flexible framework. This tool collects data and required information from existing experimental models as a source and by using the fuzzy law such as "if then else rules" and provides effective models to analyze image in better way.

\section{References}

[1] D.Selvaraj, R.Dhanasekaran, "Mri Brain Image Segmentation Techniques - A Review" Indian Journal of Computer Science and Engineering (IJCSE). Vol. 4 No.5 Oct-Nov 2013.

[2] Ibtihal D. Mustafa, Mawia A. Hassan, " A Comparison between diferrent segmentation techniques used in Medical Image", American journal of biomedical engineering 2016.

[3] Dingding Liu, Bilge Soran, Gregg Petrie, and Linda Shapiro, , "A Review of Computer Vision Segmentation Algorithms", University of Washington, 2011.

[4] A. Anand, H. Kaur, "Survey on Segmentation of Brain Tumor: A Review of Literature", IJARCCE, Vol. 5, Issue 1, January 2016.

[5] H. Salome Hema Chitra, S. Sugunal and S. Naganandini Sujatha, "A Survey on Image Analysis Techniques in Agricultural Product”, Indian Journal of Science and Technology, Vol 9(12), March 2016.

[6] Mohammed Sabbih Hamoud Al-Tamimi \& Ghazali Sulong, "A New Method for Detecting Cerebral Tissues Abnormality in Magnetic Resonance Images”, Canadian Center of Science and Education, Vol. 9, No. 8; 2015. 
[7] Saurabh Shah and N.C. Chauhan, "Techniques for Detection and Analysis of Tumours from Brain MRI Images: A Review”, JBEMi, 14th January 2016.

[8] Meena A, Raja K, "K-Means Segmentation of Alzheimer's Disease In Pet Scan Datasets An Implementation", 2010.

[9] Yashwanti Sahu, Suresh Gawande, "K MEAN and FUZZY clustering algorithm predicated brain tumor segmentation and area estimation”, IRJET, Volume: 03 Feb-2016.

[10] Nivedha.R, Mrs.Angayarkanni.N, M.E,(Ph.D.), "Brain MRI segmentation and tumor detection using FCM and NEURAL Networks”, IJARBEST, Vol. 1, May 2015.

[11] Rasel Ahmmed and Md. Foisal Hossain, "Tumor Stages Detection in Brain MRI Image using Template based K-means and Fuzzy C-means Clustering Algorithm”, 18 - 19 December, 2015, BIAM Foundation, Dhaka, Bangladesh.

[12] Asmatullah Chaudhry, Mehdi Hassan, Asifullah Khan, "Robust segmentation and intelligent decision system for cerebrovascular disease", Medical \& Biological Engineering \& Computing · April 2016.

[13] Ali Salem Bin Samma and Rosalina Abdul Salam, "Adaptation of K-Means Algorithm for Image Segmentation”, World Academy of Science, Engineering and Technology 502009.

[14] G.Santhosh Krishnan, K.Sivanarulselvan, P.Betty, "Survey on brain tunour detection and ckassification using image processing”, ISSN: Volume 2 (2016). 